\title{
Bilateral pulmonary emboli
}

\author{
Ori Galante, ${ }^{1}$ Nilly Lador, ${ }^{2}$ Dan Schwarzfuchs ${ }^{2}$
}

${ }^{1}$ Medical Intensive Care Unit, Soroka University Medical Center, Beer-Sheva, Israel ${ }^{2}$ Department of Medicine, Soroka University Medical Center, Beer-Sheva, Israel

\section{Correspondence to} Dr Ori Galante, galante1@zahav.net.il
To cite: Galante 0 , Lador N, Schwarzfuchs D. BMJ Case Reports Published online: 17 December 2012 doi:10.1136/bcr-2012007827

\section{DESCRIPTION}

A 79-year-old woman presented with an aggravating effort dyspnoea during the preceding week. On examination, her oxygen saturation was $87 \%$ while breathing ambient air; her blood pressure was 120/75; she had distended neck veins; normal breath sounds and regular heart sounds. Her liver was mildly enlarged. She had no leg oedema, swelling or tenderness. Her laboratory results were within normal limits, including troponin level. One month before admission, while cleaning her house, she fell and broke her left humerus neck. Her chest roentgenogram revealed a fracture in the surgical head of the left humerus and bilateral enlargement of the pulmonary arteries (figure 1). Chest CT angiography ascertained the suspected diagnosis of large bilateral pulmonary emboli (figure 2). Echo Doppler examination revealed left subclavian deep vein thrombosis. The patient received anticoagulation treatment as indicated for 6 months.

A fractured limb is a risk factor for venous thromboembolism, bilateral hillar enlargement can be due to pulmonary arteries enlargement which is a roentgenogramic sign of pulmonary hypertension (Palla's sign). These findings, along with lung oligemia (Westermark's sign) suggest the diagnosis.

Approximately $1 \%$ to $4 \%$ of all DVT cases involve the upper extremity, with $3 \%$ to $14 \%$ of these cases complicated by pulmonary embolism, a

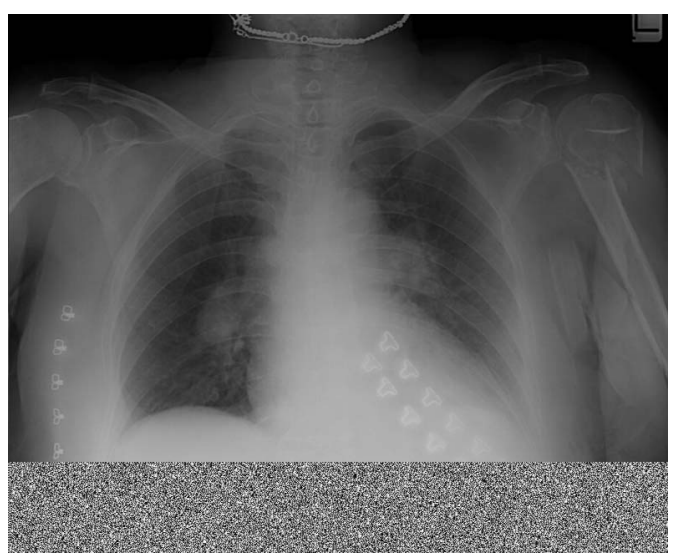

Figure 1 Chest roentgenogram showing a fracture in the surgical head of the left humerus, bilateral enlargement of the pulmonary arteries and bilateral pulmonary oligemia.

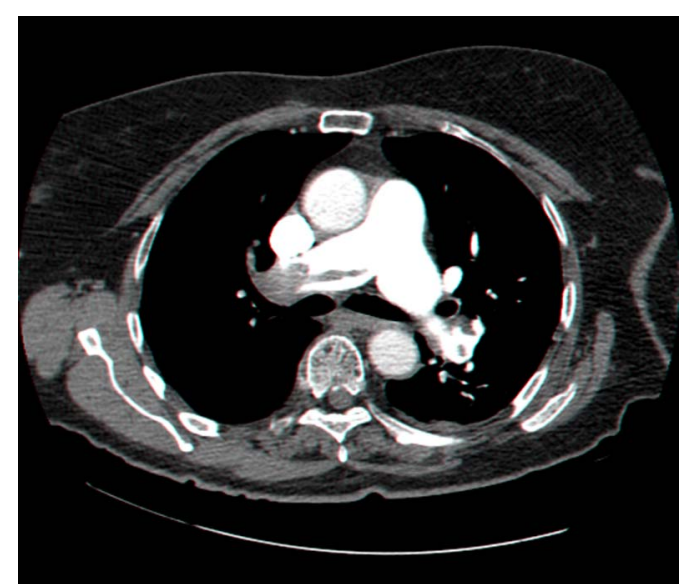

Figure 2 Chest CT angiography showing bilateral pulmonary emboli.

considerably lower incidence compared to that of lower extremity (which is between 16\% and 29\%).

Upper extremity deep venous thrombosis is either primary (idiopathically or effort-related), or secondary - most commonly malignancy or central venous catheter related. ${ }^{1}$ A rare risk factor is an upper extremity fracture. ${ }^{2}$

\section{Learning points}

A fractured immobilised limb is a risk factor for deep vein thrombosis.

- Chest roentgenogram examination should start with soft and bony tissue before inspecting the heart and lung. A thorough inspection of the surrounding hard and soft tissue, focusing eventually on the enlarged lung hilus, can help establish the correct diagnosis in this case.

Competing interests None.

Patient consent Obtained.

\section{REFERENCES}

1 Czihal M, Hoffmann U. Upper extremity deep venous thrombosis. Vasc Med 2011;16:191-202.

2 Sawyer GA, Hayda R. Upper-extremity deep venous thrombosis following humeral shaft fracture. Orthopedics 2011;34:141. 